\title{
Zur Physiologie der Oxalsäure und Oxalursäure im Harn.
}

Von

Dr. A. M. Luzzatto, Privatdocent in Padua.

(Aus dem chemischen Laboratorium des pathologischen Instituts zu Berlin.)

(Der Redaction zugegangen am 26. December 1902.)

E. Schunck ${ }^{1}$ ) hat schon im Jahre 1867 auf die Anwesenheit von präformirter 0xalursäure im Harn hingewiesen; er konnte oxalursaures Ammon, wenn auch nur in sehr geringer Menge, aus 100 Liter Urin darstellen, die er langsam auf Thierkohle tropfen liess; durch Extraction der Kohle mit Alkohol gewann er eine krystallinische Verbindung, welche in ihren chemischen Eigenschaften mit dem aus Parabansäure erhaltenen oxalursauren Ammon vollständig übereinstimmte. Durch eine Nachprüfung von Neubauer ${ }^{2}$ ) wurde der Befund bestätigt. Man konnte aber seinen Nachweis nicht für ganz einwandfrei halten, da die Möglichkeit nicht ausgeschlossen war, dass die Oxalursäure nicht präformirt war, sondern sich bei dem langdauernden Filtriren auf Kohle gebildet hatte. Die Frage blieb so jahrelang unentschieden, bis sie im Jahre 1900 von E. Salkowski ${ }^{3}$ ) wieder aúfgenommen wurde. Er fand nämlich, dass Harn nach starker Concentrirung und nachfolgendem Zusatz von Salzsäure erheblich mehr Oxalsäure lieferte als eine Probe desselben Urins, welche man ohne Säurezusatz concentrirt hatte; eine Thatsache, die sich am besten durch die Annahme der Abstammung der Oxalsäure aus einer in Aether unlöslichen Verbindung, welche durch Salzsäure ge- 
spalten wird (sehr wahrscheinlich Oxalursäure), erklären liess. Salkowski beobachtete ausserdem, dass mehrere Urine, die er untersuchte, trotz Concentrirung und dreimaliger Ausschüttelung mit Aether, bei nachfolgendem Kochen mit Salzsäure noch verhältnissmässig bedeutende Mengen von Oxalsäure lieferten. Wie er selbst aber ausdrücklich betont, konnte man auch seine Versuche nicht als ganz einwandfrei betrachten, da einige Nachuntersuchungen die Möglichkeit nachgewiesen hatten, dass die Oxalsäure nach längerem Kochen oder Eindampfen in eine in Aether unlösliche, später durch Kochen mit Salzsäure abspaltbare Verbindung übergeht. Es war also hier auch nicht ausgeschlossen, dass die Oxalursäure nicht präformirt, sondern nur bei der Bearbeitung gebildet wäre. Daraus folgt, dass, wenn man jede Fehlerquelle bei der Bestimmung der präformirten und der abgespaltenen Oxalsäure vermeiden will, dass man dann die erstere in von Natur aus concentrirten, aber nicht eingedampften Urinproben bestimmen muss; der Werth der Oxalursäure wird durch die Differenz zwischen der Menge der gesammten Oxalsäure nach Kochen mit Salzsäure und der Menge der präformirten Oxalsäure ausgedrückt.

Nach dieser Richtung habe ich auf Anregung von Herrn Professor Salkowski die folgenden Versuche angestellt, um womöglich endgültig die Frage über die Anwesenheit von abspaltbarer Oxalsäure (die ich der Kürze wegen Oxalursäure nennen will) im Urin $\mathrm{zu}$ entscheiden.

Meine Bestimmungen wurden nach der schon allgemein bekannten Methode von Salkowski (Ausschüttelung mit Aetheralkoholmischung) durchgeführt.*)

*) Barth und Autenrieth4) haben neulich eine Methode der Oxalsäurebestimmung angegeben, welche die ursprüngliche Salkowskische Methode insofern modificirt, als die Aetherausschüttelungen nicht direct im Urin ausgeführt werden; es wird vielmehr die Oxalsäure mit Ammoniak und Chlorcalcium gefällt; der Niederschlag wird mit Salzsäure gelöst und mit der Aetheralkoholmischung ausgeschüttelt; im Uebrigen verfährt man nach den Salkowski'schen Angaben. Ich habe die Methode mit der ursprünglichen von Salkowski verglichen und folgendè Resultate bekommen: 
Ich habe mich aber niemals mit 3 Ausschüttelungen begnügt, sondern immer bis zu völliger Erschöpfung des Harns ausgeschüttelt. Im Allgemeinen habe ich jeden Urin $9 \mathrm{Mal}$ mit je $180 \mathrm{ccm}$ Aether und $20 \mathrm{ccm}$ Alkohol ausgeschüttelt, unter Trennung von je 3 Ausschüttelungen von untereinander; nur wenn ich aus den 3 letzten keine oder nur Spuren von Oxalsäure erhielt, war die Bestimmung als beendigt zu betrachten. Ich bin in der Weise verfahren, weil ich mich leicht überzeugen konnte, dass, speciell wo man, wie ich, mit nicht concentrirten Harnen arbeitet, es oft ganz unmöglich ist, den Urin mit 3 Ausschüttelungen zu erschöpfen. Das umsomehr, als die Oxalsäure bei einigen Urinen leicht, bei anderen schwerer in Aether übergeht, ohne dass man im Stande ist, die verschiedenen Bedingungen genau zu ermitteln. Es ist wohl möglich, dass die Oxalsäure, wie Barth und Auten-

$200 \mathrm{ccm}$ Hundeharn liefern:

a) nach Salkowski 0,0041 g Oxalsäure,

b) nach Barth und Autenrieth 0,0044 g Oxalsäure.

Man kann also die zwei Methoden als gleichwerthig bezeichnen, umsomehr als ich mich überzeugen konnte, dass das Filtrat nach der Fällung mit Chlorcalcium und Ammoniak keine Oxalsäure enthält. Es fallen aber auch die Einwände fort, welche Barth und Autenrieth gegen die Salkowski'sche Methode hervorgehoben haben, nämlich, dass es unmöglich ist, die Oxalsäure aus verdünnten Lösungen vollständig zu extrahiren. Es sprechen dagegen alle nachfolgenden Zahlen, sowie die vielen Untersuchungen, welche von mehreren Seiten nach dieser Methode ausgeführt worden sind. Ich war nur im Stande, zu constatiren, dass die Oxalsäure aus sehr verdünnten Lösungen sich nur dann ausschütteln lässt, wenn die betreffende Flüssigkeit stark mit Salzsäure angesäuert wird, ein Umstand, welcher vielleicht die negativen Resultate von Barth und A utenrieth erklären kann.

Bei meinen Untersuchungen habe ich den Eindruck gewonnen, dass bei dieser neuen Methode die Ausschüttelung vielleicht schnellẹ als bei der ursprünglichen erfolgt; das trifft aber nur für an Oxalsäure verhältnissmässig reiche Urine zu. Trotzdem habe ich mich fast immer der Salkowski'schen Methode bedient, einerseits weil ich meine Resultate mit denen von Salkowski vergleichen wollte, andererseits weil eine Fällung der Oxalursäure als Calciumoxalurat nicht absolut von der Hand zu weisen war; nur bei einer etwas verschiedenen Versuchsanordnung habe ich mich, wie später angeführt sein wird, auch dieser Methode bedient. 
rieth annehmen, sich leichter aus concentrirten Lösungen ausschütteln lässt; das trifft aber nicht für alle Fälle zu; z. B. konnte ich einen menschlichen Harn, welcher auf $200 \mathrm{ccm}$ nur $0,0012 \mathrm{~g}$. Oxalsäure enthielt, mit dreimaligen Ausschüttelungen völlig erschöpfen, während bei einem conservirten Hundeharne, welcher auf $200 \mathrm{ccm} 0,1385 \mathrm{~g}$ Oxalsäure enthielt, die Bestimmung erst nach 18(!) Ausschüttelungen fertig war. Ich halte es nicht für unmöglich, dass wir eigenthümliche, ganz unbekannte individuelle Bedingungen vor uns haben, weil, soviel ich sehen konnte, Urin eines und desselben Individuums in dieser Beziehung sich immer ungefähr in ein und derselben Weise verhält; z. B. war der letztgenannte Urin immer sehr schwer auszuschütteln. Auch nachdem er zu anderen Zwecken von seiner Oxalsäure durch Fällung befreit und mit käuflicher Oxalsäure versetzt war, konnte man dieselbe nur mit 12 Ausschüttelungen vollständig extrahiren. Ich muss auch dazu hinzufügen, dass die totale Erschöpfung etwas schneller an gekochten als an nicht gekochten Urinen gelang, ohne dass ich im Stande bin, zu sagen, worauf das beruht.

Nach dieser Methode habe ich Parallelbestimmungen an verschiedenen Urinen in folgender Weise ausgeführt: $200 \mathrm{ccm}$ Urin wurden mit $20 \mathrm{ccm} 25 \%$ iger Salzsäure angesäuert und direct (um die von Salkowski angenommene mögliche Umwandlung der Oxalsäure in Oxalursäure auszuschliessen) bis zur völligen Erschöpfung ausgeschüttelt (die Extraction dauerte nie mehr als $1 \frac{1 / 2}{2}$ Stunde). Weitere $200 \mathrm{ccm}$ waren gleichzeitig mit $40 \mathrm{ccm}$ derselben Salzsäure versetzt, eine halbe bis dreiviertel Stunde (nach dem Beginn des Siedens) auf dem Drahtnetz im Kolben gekocht und nach völligem Erkalten in derselben $\mathrm{W}^{T}$ eise extrahirt. Nach diesem Verfahren war jede Möglichkeit einer Umwandlung der präformirten Oxalsäure in eine unlösliche Form ausgeschlossen und folglich war jedes eventuelle Plus an Oxalsäure im gekochten, stark angesäuerten Harne der abspaltbaren Form, also höchst wahrscheinlich der Oxalursäure zuzuschreiben. Ich konnte thatsächlich in verschiedenen Harnen diese abspaltbare Oxalsäure nachweisen, wie es aus folgenden Zahlen erhellt: 
Tabelle I.

\begin{tabular}{|c|c|c|c|c|c|c|c|c|}
\hline & \multicolumn{3}{|c|}{ Ungekochter Harn } & \multicolumn{3}{|c|}{$\begin{array}{c}\text { Mit HCl gekochter } \\
\text { Harn }\end{array}$} & \multirow{2}{*}{$\begin{array}{c}\text { Abspalt- } \\
\text { bare } \\
\text { Oxal- } \\
\text { säure } \\
\text { auf } \\
1000 \mathrm{ccm}\end{array}$} & \multirow{2}{*}{$\begin{array}{c}\text { Ver- } \\
\text { hältniss } \\
\text { der ab- } \\
\text { spaltbaren } \\
\text { Oxalsäure } \\
\text { zur } \\
\text { Gesammt- } \\
\text { menge }\end{array}$} \\
\hline & $\mathrm{CaO}$ & $\left|\begin{array}{c}\text { Oxal- } \\
\text { säure } \\
\text { auf } \\
200 \mathrm{ccm}\end{array}\right|$ & $\begin{array}{c}\text { Oxal- } \\
\text { säure } \\
\text { auf } \\
1000 \mathrm{ccm}\end{array} \mid$ & $\mathrm{CaO}$ & $\left|\begin{array}{c}\text { Oxal- } \\
\text { säure } \\
\text { auf } \\
200 \mathrm{ccm}\end{array}\right|$ & \begin{tabular}{|c|} 
Oxal- \\
säure \\
auf \\
$1000 \mathrm{ccm}$
\end{tabular} & & \\
\hline $\begin{array}{c}1 . \\
\text { Conservirter } \\
\text { Hundeharn }\end{array}$ & 0,0403 & 0,0647 & 0,3235 & 0,0483 & 0,0776 & 0,3880 & 0,0645 & $1 / 6$ \\
\hline $\begin{array}{c}2 . \\
\text { Frischer } \\
\text { Hundeharn }\end{array}$ & 0,0026 & 0,0041 & 0,0205 & 0,0052 & 0,0083 & 0,0415 & 0,0210 & $1 / 2$ \\
\hline $\begin{array}{c}3 . \\
\text { Frischer } \\
\text { Hundeharn }\end{array}$ & 0,0032 & 0,0051 & 0,0255 & 0,0050 & 0,0080 & 0,0400 & 0,0145 & $\begin{array}{c}\text { etwas mehr } \\
\text { als } 1 / 3\end{array}$ \\
\hline $\begin{array}{c}4 . \\
\text { Frischer } \\
\text { Hundeharn }\end{array}$ & 0,0024 & 0,0038 & 0,0190 & 0,0059 & 0,0094 & 0,0470 & 0,0280 & $\begin{array}{c}\text { mehr als } \\
1 / 2\end{array}$ \\
\hline $\begin{array}{c}5 . \\
\text { Frischer } \\
\text { Hundeharn }\end{array}$ & 0,0054 & 0,0086 & 0,0430 & 0,0064 & 0,0102 & 0,0510 & 0,0080 & $\begin{array}{c}\text { etwas weniger } \\
\text { als } 1 / 6\end{array}$ \\
\hline $\begin{array}{c}6 . \\
\text { Conservirter } \\
\text { Kaninchen- } \\
\text { harn }\end{array}$ & 0,0094 & 0,0102 & 0,0510 & 0,0068 & 0,0109 & 0,0545 & 0,0035 & $\begin{array}{c}\text { etwas weniger } \\
\text { als } 1 / 1 \text { s }\end{array}$ \\
\hline $\begin{array}{c}7 . \\
\text { Frischer Ka- } \\
\text { ninchenharn }\end{array}$ & 0,0004 & 0,0006 & 0,0030 & 0,0016 & 0,0025 & 0,0125 & 0,0095 & $\begin{array}{c}\text { etwas weniger } \\
\text { als } \% / 8\end{array}$ \\
\hline $\begin{array}{l}\quad 8 . \\
\text { Frischer Ka- } \\
\text { ninchenharn }\end{array}$ & $\begin{array}{l}\text { Tuwás } \\
\text { bare } \\
\text { Spuren }\end{array}$ & - & 一 & 0,0011 & 0,0017 & 0,0085 & 一 & - \\
\hline
\end{tabular}

Nach den vorangehenden Zahlen scheint es mir also einwandfrei bewiesen, dass eine durch Kochen mit Salzsäure abspaltbare Form der Oxalsäure im Urin vorhanden ist. Dieselbe ist aber nicht in sämmtlichen Urinen anwesend, sondern auch bei einem und demselben Thiere constatirte ich sie nur gelegentlich, ohne die Bedingungen ihres Auftretens feststellen 
zu können. Auch ihre absolute und relative Menge zeigte, wie aus den mitgetheilten Zahlen hervorgeht, grosse Schwankungen (von $1 / 9-7 / 8$ der Gesammtmenge).

Im menschlichen Urin konnte ich die abspaltbare 0xalsäure nur einmal in sehr kleinen Mengen und nach folgendem Verfahren nachweisen, wie aus dem folgenden Versuche erhellt:

$950 \mathrm{ccm}$ von frischem menschlichen Urin werden (nach Barth und Autenrieth) mit Ammoniak und Chlorcalcium gefällt und nach 24stündigem Stehen filtrirt. Der Niederschlag wird in verdünnter Salzsäure gelöst und $6 \mathrm{Mal}$ mit Alkoholäthermischung extrahirt. Man erhält daraus:

$$
0,0060 \mathrm{~g} \mathrm{CaO}=0,0096 \mathrm{~g} \text { Oxalsäure. }
$$

Das Filtrat wird mit $100 \mathrm{ccm}$ Salzsäure versetzt und bis zu ungefähr $200 \mathrm{ccm}$ concentrirt und nachher auch $6 \mathrm{Mal}$ extrahirt. Die 6 Ausschüttelungen liefern:

$$
0,0010 \mathrm{~g} \mathrm{CaO}=0,0016 \mathrm{~g} \text { Oxalsäure. }
$$

$\mathrm{Da}$ von mir auch, wie oben angeführt, bestätigte Versuche von Barth und Autenrieth bewiesen haben, dass sich keine Oxalsäure im Filtrat (nach der Fällung mit Chlorcalcium) nachweisen lässt, so müssen wir annehmen, dass die darin gefundene Oxalsäure sich durch das Eindampfen mit Salzsäure abgespalten habe; ich glaube also, auch damit einen neuen Beweis der Anwesenheit von präformirter Oxalursäure im Urin geliefert $\mathrm{zu}$ haben. Das erhellt in noch klarerer Weise aus einem Versuche, den ich an Hundeharn angestellt habe. Dabei fand ich auf $500 \mathrm{ccm}$ im Niederschlage:

im Filtrat:

$$
0,0066 \mathrm{~g} \mathrm{CaO}=0,0106 \mathrm{~g} \text { Oxalsäure, }
$$

$$
0,0022 \mathrm{~g} \mathrm{CaO}=0,0037 \mathrm{~g} \text { Oxalsäure. }
$$

Es ist mir sehr schwer gewesen, das Vorhandensein dieșer abspaltbaren Oxalsäure auf einem anderen Wege nachzuweisen; es war nämlich meine Absicht, eine bestimmte Portion Harn kalt bis zur völligen Erschöpfung mit Salzsäure auszuschütteln, nachher den Urin unter nachträglichem Zusatz von Salzsäure zu kochen und aus dem gekochten Harne wieder Oxalsäure zu erhalten. Das ist mir nur theilweise und indirect gelungen, wie aus folgenden Zahlen ersichtlich ist:

$200 \mathrm{ccm}$ Hundeharn werden mit $20 \mathrm{ccm}$ Salzsäure versetzt und 9 Mal, ohne zu kochen, extrahirt; die 3 ersten Ausschüttelungen liefern 
$0,0142 \mathrm{~g} \mathrm{CaO}=0,0228 \mathrm{~g}$ Oxalsäure; die 4., 5., 6. Aussch üttelung $0,0112 \mathrm{~g} \mathrm{CaO}=0,0180 \mathrm{~g}$ Oxalsäure; die 7., 8., 9. Ausschüttelung $0,0056 \mathrm{~g} \mathrm{CaO}=0,0090 \mathrm{~g}$ Oxalsäure; eine ganz gleiche Portion desselben Harnes wird nach Ansäuern mit $20 \mathrm{ccm}$ Salzsäure $6 \mathrm{Mal}$ in der gleichen Weise extrahirt; die 3 ersten Ausschüttelungen liefern $0,0140 \mathrm{~g}$ $\mathrm{CaO}=0,0225 \mathrm{~g}$ Oxalsäure ; die 4., 5., 6. Ausschüttelung liefern $0,0110 \mathrm{~g}$ $\mathrm{CaO}=0,0176 \mathrm{~g}$ Oxalsäure. Der so $6 \mathrm{Mal}$ ausgeätherte Urin wird jetzt mit weiteren $20 \mathrm{ccm}$ Salzsäure versetzt und $9 / 4$ Stunde kochen gelassen; 3 weitere Ausschüttelungen liefern $0,0102 \mathrm{~g} \mathrm{CaO}=0,0163 \mathrm{~g}$ 0xalsäure, also fast das Doppelte als in ungekochtem Harne.

Es ist mir aber trotz wiederholter Versuche niemals gelungen, diese Thatsache an einem kalt völlig erschöpften Urin zu constatiren. Worauf das beruht, kann ich nicht mit voller Bestimmtheit sagen; ich muss aber hervorheben, dass sämmtliche erschöpfte Proben mehrere Tage hindurch stehen geblieben waren; es scheint also nicht unwahrscheinlich, dass die abspaltbare Oxalsäure bei der Anwesenheit von Salzsäure in eine in Aether unlösliche Verbindung übergeht. Diese Annahme wird, so viel ich glaube, von folgendem Versuche wesentlich unterstützt:

Der kalt völlig extrahirte Hundeharn vom Versuche 4 wird mit weiteren $20 \mathrm{ccm}$ Salzsäure versetzt und eine halbe Stunde lang gekocht; am folgenden Tage wird er noch $3 \mathrm{Mal}$ extrahirt; man erhält daraus $0,0004 \mathrm{~g} \mathrm{CaO}=0,0006 \mathrm{~g}$ Oxalsäure, also eine minimale Menge im Vergleich mit der des direct gekochten Harnes. Der Urin wird nach einigen Tagen mit Ammoniak alkalisirt und mit Chlorcalcium gefällt; der Niederschlag wird auf einem Filter gesammelt und in verdünnter Salzsäure gelöst. Man concentrirt die etwas zu reichliche Lösung, welche nachher filtrirt wird, um die ausgeschiedenen harzigen Substanzen, die sie enthält, zu entfernen. Dann fällt man wieder mit Ammoniak und säuert mit Essigsäure an. Der Niederschlag enthält neben organischen Substanzen auch zahlreiche, wohlausgebildete Krystalle von oxalsaurem Kalk. Man erhält daraus (nach Abziehung des mitgefällten Eisenoxyds)*) 0,0064 g $\mathrm{CaO}=0,0102 \mathrm{~g}$ 0xalsäure, also etwas mehr als in direct gekochtem Harne.

*) $\mathrm{Zu}$ diesem Zwecke wurde die im Tiegel abgewogene Asche in verdünnter Salzsäure gelöst, die Lösung mit Ammoniak alkalisirt, mit Natriumphosphat versetzt und mit Essigsäure angesäuert. Nach etwa 2 Stunden wurde der aus Ferriphosphat bestehende Niederschlag abfiltrirt und verascht. 
Jetzt frägt es sich nun, ob die abspaltbare Form der Oxalsäure mit der Oxalursäure zu identificiren ist. Das ist von vornherein sehr wahrscheinlich, da wir keine andere Verbindung ausser der Oxalursäure kennen, welche, mit Salzsäure gekocht, Oxalsäure liefern kann. Zweitens konnte ich ein ganz analoges Verhalten nach Zusatz von oxalursaurem Ammon*) zu Urin constatiren, wie es aus folgendem Versuche erhellt:

$400 \mathrm{ccm}$ von frischem menschlichen Urin werden mit $0,28 \mathrm{~g}$ oxalursauren Ammons versetzt, bis zu völliger Lösung geschüttelt und in zwei gleiche Theile getheilt. Der erste Theil wird mit $20 \mathrm{ccm}$ Salzsäure angesäuert und sofort $9 \mathrm{Mal}$ ausgeschüttelt. Nach 4 Tagen wird der Urin mit weiteren $20 \mathrm{ccm}$ Salzsäure versetzt, eine halbe Stunde lang gekocht und $6 \mathrm{Mal}$ wieder extrahirt. Der zweite Theil wird sogleich mit $40 \mathrm{ccm}$ Salzsäure angesäuert, gekocht und bis zu völliger Erschöpfung ausgeschüttelt. Daraus erhält man folgende Werthe:

Tabelle II.

\begin{tabular}{|c|c|c|}
\hline & $\mathrm{CaO}$ & Oxalsäure \\
\hline Aus dem ungekochten Harne $\ldots \ldots \ldots$ & 0,0065 & 0,0105 \\
\hline $\begin{array}{l}\text { Aus dem kalt erschöpften und nachher mit } \\
\text { HCl gekochten Harne } \ldots \ldots \ldots\end{array}$ & 0,0058 & 0,0092 \\
\hline Aus dem mit $\mathrm{HCl}$ gekochten Harne & 0,0101 & 0,0161 \\
\hline
\end{tabular}

Aus diesem Versuche sind, meiner Meinung nach, zwei Umstände hervorzuheben. Zuerst lässt sich hier die zugesetzte Oxalursäure sehr leicht und völlig**) aus dem ausgeätherten Harne durch Kochen extrahiren, während, wie gesagt, das im natürlichen Harne nicht vorzukommen pflegt. Es ist sehr schwer, diesen Umstand zu erklären; man könnte vielleicht entweder an den Unterschied in der Menge der Substanz, oder

*) Die Substanz wurde durch Lösung von käuflicher Parabansäure (Kahlbaum) in ammoniakalischem Wasser gewonnen.

**) 0,28 g oxalursaures Ammon entsprechen 0,169 g Oxalsäure, also etwas weniger als die von mir gefundene Menge Oxalsäure, was sich durch den natürlichen Gehalt an Oxalsäure des Urins leicht erklären lässt. 
an eigenthümliche Verbindungen denken. Worauf der übrigens sehr kleine Unterschied zwischen dem direct gekochten und dem nach Ausätherung gekochten Harne beruht, konnte ich nicht mit Sicherheit eruiren.

Zweitens wird ein erheblicher Theil der Oxalursäure durch das einfache Ansäuern des Urins in Oxalsäure verwandelt. Diese Umwandlung wurde schon von Schunck hervorgehoben und ich konnte seine Angaben völlig bestätigen. Wenn man eine wässerige Lösung von oxalursaurem Ammon mit Salzsäure ansäuert und mit Ammoniak und Chlorcalcium versetzt, so bildet sich im Laufe von wenigen Minuten ein Niederschlag, welcher aus schönen grossen Oktaedern*) besteht. Die Umwandlung ist aber keine vollständige, da man in der filtrirten Flüssigkeit durch Kochen einen neuen Niederschlag bekommt. Im Urin kann man unter Umständen durch einfaches Ansäuern ohne Weiteres einen Niederschlag von oxalsaurem Kalk bekommen. Sehr kleine Mengen von oxalursaurem Ammon können auch, wie ich mich überzeugen konnte, durch Ansäuern völlig in Oxalsäure umgewandelt werden. Folglich besteht die Möglichkeit, dass ein viel grösserer Theil der Oxalsäure, als man jetzt das Recht anzunehmen hat; als Oxalursäure ausgeschieden wird; ein Umstand, welcher viel theoretisches und praktisches Interesse darbieten könnte, weil man damit viele dunkle Verhältnisse bezüglich der Löslichkeit der Oxalsäure im Urin zu erklären im Stande wäre. Leider sind wir, soviel ich weiss, nicht im Stande, einen Beweis dafür zu liefern, weil es ganz unmöglich ist, ohne starkes Ansäuern die Oxalsäure aus dem Urin mit Aether zu extrahiren. Auch eine indirecte Methode (wie z. B. die etwaige Bestimmung der Vermehrung des Harnstoffes, welcher aus der Spaltung der Oxalursäure entsteht) wäre der geringen Mengen wegen völlig unbrauchbar.

Schunck hat der Anwesenheit von präformirter Oxalursäure im Urin grosses Gewicht beigelegt, weil er damit die

*) Der Niederschlag, welcher nach der Umwandlung der Oxalursäure durch das Kochen entsteht, bildet dagegen in der Regel kleine, langgezogene sechsseitige rhombische Täfelchen. 
etwas räthselhafte langsame Fällung des Calciumoxalats im Urin vollständig erklärt glaubte. Seine Meinung scheint mir aber nicht ganz einwandfrei zu sein, wie aus folgenden Versuchen erhellt: Mehrere menschliche Urine verschiedener Provenienz wurden mit oxalursaurem Ammon in verschiedener Menge versetzt und tage- bis monatelang mit und ohne Chloroformzusatz bei Zimmertemperatur stehen gelassen. In keinem Falle, auch nach mehreren Monaten, konnte ich einen erheblichen Niederschlag von Calciumoxalat wahrnehmen. Wo einige spärliche Krystalle vorhanden waren, konnte man ungefähr dieselbe Menge auch in der Controllprobe constatiren; nirgends waren zwischen Urinprobe mit Oxalurat und Controllprobe so erhebliche Unterschiede vorhanden, dass die Annahme einer Bildung von Oxalsäure aus Oxalursäure gerechtfertigt erscheinen könnte. Folglich muss man entweder annehmen, dass die natürlichen Verhältnisse von den experimentellen gründlich verschieden sind, oder die Schunck'sche Hypothese vollständig zurückweisen.

Salkowski hat schon gelegentlich bemerkt, dass lange Zeit mit Chloroform conservirte Harne auffallend wenig Harnsäure enthalten, und auf die Möglichkeit hingewiesen, dass die Harnsäure unter Bildung von Oxalsäure zersetzt sein könne. Im Laufe dieser Untersuchungen war ich im Stande, diese Annahme $\mathrm{zu}$ bestätigen. Ein Hundeharn, welcher seit langer Zeit mit Chloroform conservirt war, lieferte (kalt extrahirt) durch 18 Ausschüttelungen am 28. Mai 1902 0,0403 g CaO = 0,0647 g Oxalsäure. Derselbe Urin (ohne dass man eine Veränderung des specifischen Gewichtes wahrnehmen konnte), in derselben Weise $18 \mathrm{Mal}$ am 3. September 1902 extrahirt, lieferte $0,0862 \mathrm{~g} \mathrm{CaO}=0,1385 \mathrm{~g}$ Oxalsäure.

$\mathrm{Ob}$ thatsächlich die Oxalsäure sich aus der Harnsäure abgespalten hat, muss ich dahingestellt lassen, da ich keine Harnsäurebestimmungen vorgenommen habe. Jedenfalls ist die Frage nicht ohne theoretisches Interesse und wird vielleicht Gegenstand-von künftigen Untersuchungen sein.

Bei der Ausführung der Parallelbestimmungen von Oxalsäure in ungekochten und mit Salzsäure gekochten Urinen war 
meine Aufmerksamkeit auf eine Thatsache gelenkt worden, welche für die genaue Bestimmung der Oxalsäure viel Interesse darbieten kann. In mehreren Harnen fand ich nämlich, dass der Gehalt an Oxalsäure kleiner nach als vor dem Kochen war; das kann man leicht aus folgenden Zahlen ersehen:

Tabelle III

(sämmtliche Zahlen sind auf $1000 \mathrm{ccm}$ berechnet).

\begin{tabular}{|c|c|c|c|c|}
\hline & \multicolumn{2}{|c|}{ Ungekochter Harn } & \multicolumn{2}{|c|}{ Mit HCl gekochter Harn } \\
\hline & $\mathrm{CaO}$ & Oxalsäure & $\mathrm{CaO}$ & Oxalsäure \\
\hline $\begin{array}{c}1 . \\
\text { Conservirter } \\
\text { Hundeharn }\end{array}$ & 0,4310 & 0,6925 & 0,3300 & 0,5300 \\
\hline $\begin{array}{c}2 . \\
\text { Frischer } \\
\text { Menschenharn }\end{array}$ & 0,0070 & 0,0110 & 0,0040 & 0,0060 \\
\hline $\begin{array}{c}3 . \\
\text { Frischer Menschenharn } \\
\text { von demselben Individ. } \\
\text { vom Versuche } 2\end{array}$ & 0,0170 & 0,0270 & 0,0110 & $\begin{array}{c}0,0175 \\
.\end{array}$ \\
\hline $\begin{array}{c}4 . \\
\text { Frischer } \\
\text { Hundeharn }\end{array}$ & 0,0240 & 0,0385 & 0,0185 & 0,0295 \\
\hline $\begin{array}{c}\mathbf{5} . \\
\text { Frischer } \\
\text { Kaninchenharn }\end{array}$ & 0,0110 & 0,0176 & 0,0051 & 0,0081 \\
\hline
\end{tabular}

Diese Versuche stehen nicht ganz isolirt da, da Barth und Autenrieth auf die Möglichkeit einer Zerstörung der Oxalsäure durch längeres Kochen hinweisen. Hildebrand ${ }^{5}$ ) konnte auch eine sehr erhebliche Verminderung von relativ 
grossen Quantitäten Oxalsäure bei einstündigem Kochen mit Salzsäure beweisen. Ich habe mehrmals Oxalsäure zu Urin zugesetzt, einen Theil vor und einen Theil nach dem Kochen extrahirt und immer ganz übereinstimmende Werthe gefunden; ebensowenig konnte ich in wässerigen Lösungen von Oxalsäure durch Kochen mit Salzsäure eine Zerstörung constatiren. Worauf der Unterschied zwischen diesen und den vorhergehenden Versuchen beruht, ist sehr schwer zu sagen, und ebenso ist es nicht leicht, die Verminderung der Oxalsäure durch Kochen zu erklären. Barth und Autenrieth nehmen eine Oxydation der Oxalsäure an, eine Hypothese, welche theoretisch nicht sehr wahrscheinlich zu sein scheint. Man könnte vielleicht an fermentative Zerstörung denken, wie sie von Klemperer und Tritschler ${ }^{6}$ ) für Blut und Faeces bewiesen wurde. Da aber der Urin lange Zeit gekocht und stark angesäuert wurde, hat auch eine solche Annahme sehr wenig für sich.

Wir müssen uns folglich mit der Constatirung dieses Unterschiedes begnügen; praktisch ist es zu beachten, dass, um eine ganz genaue Oxalsäurebestimmung auszuführen, zwei Parallelbestimmungen im ungekochten und im gekochten Harne nöthig sind. Man kann nämlich nicht von vornherein wissen, ob man bei einem bestimmten Urine mehr Oxalsäure in der gekochten oder in der ungekochten Probe finden wird.

Auf Anregung von Herrn Professor Salkowski habe ich auch die schon mehrmals geprüfte Frage, ob Oxalsäure auf Harnsäure im thierischen Organismus entsteht, wieder aufgenommen. Aeltere Autoren, wie Wöhler und Frerichs, ?) hatten sowohl bei Menschen als beim Hunde, sowohl nach Fütterung als nach Einspritzung von harnsauren Salzen, einen reichlichen Niederschlag von Calciumoxalat im Urin constatirt, aber keine quantitativen Bestimmungen vorgenommen. Diese Versuche wurden theilweise von Gallois ${ }^{8}$ ) bestätigt, welcher an sich selbst nach Einführung von harnsaurem Kali einmal zahlreiche Krystalle von Calciumoxalat im Urin, einmal nichts fand. Lommel ${ }^{9}$ ) fand, dass eine nucleinreiche Diät sowohl eine Vermehrung der Harnsäure, wie der Oxalsäure im Urin 
verursacht. Lüthje, ${ }^{10}$ ) Salkowski ${ }^{11}$ ) und Stradomsky ${ }^{12}$ ) konnten aber das nicht bestätigen. Neubauer ${ }^{13}$ ) hat beim Kaninchen keine eigentliche Vermehrung der Oxalsäure nach fünftägiger Harnsäurefütterung constatirt, hat aber auch keine quantitativen Bestimmungen gemacht; er ist trotzdem geneigt, diese Umwandlung unter gewissen Umständen (Verlangsamung der Oxydationsvorgänge) anzunehmen. Ebenso Fürbringer, ${ }^{14}$ ) welcher nach Einführung von Ammoniumurat bei mehreren Individuen nur gelegentlich Vermehrung der Oxalsäureausscheidung wahrnehmen konnte. Diese Inconstanz schreibt er der verschiedenen Intensität der Oxydationsvorgänge und der verschiedenen Intensität, mit welcher Oxalursäure und Oxalsäure davon beeinflusst werden, zu. Von den neueren Autoren haben sich Klemperer und Tritschler ${ }^{15}$ ) mit der Frage beschäftigt und sind zu ganz negativen Resultaten gekommen. Ich füge noch hinzu, dass Wiener ${ }^{16}$ ) nach Fütterung mit Alloxan, einer der Harnsäure nahestehenden Substanz, Symptome von Oxalsäurevergiftung und erhebliche Vermehrung der Oxalsäure im Urin wahrnahm.

Ich habe mich mit der Frage speciell vom Standpunkte der Oxalursäureausscheidung beschäftigt; es war nämlich sehr interessant, zu erfahren, ob für die Harnsäure auch im Thierorganismus die bekannte Umwandlung in Alloxan, Parabansäure, Oxalursäure stattfindet, obwohl auch eine directe Umwandlung nicht von der Hand zu weisen ist, da Salkow ski ${ }^{17}$ ) nach Einwirkung von Ferrichlorid auf Harnsäure eine unmittelbare Spaltung in Oxalsäure und Harnstoff nachgewiesen hat.

Ich habe diesbezüglich folgende Versuche ausgeführt:

Versuch I. Ein Hund von 6,900 Kilo Gewicht wird während 15 Tagen mit $200 \mathrm{~g}$. Schabefleisch und $40 \mathrm{~g}$ Speck täglich gefüttert. Vom 6. bis zum 10. Tage werden der Nahrung 2,50 g Harnsäure täglich zugesetzt. Am Ende des Versuches wiegt das Thier 6,870 Kilo. Die Harnuntersuchung liefert folgende Werthe: 
Tabelle IV.

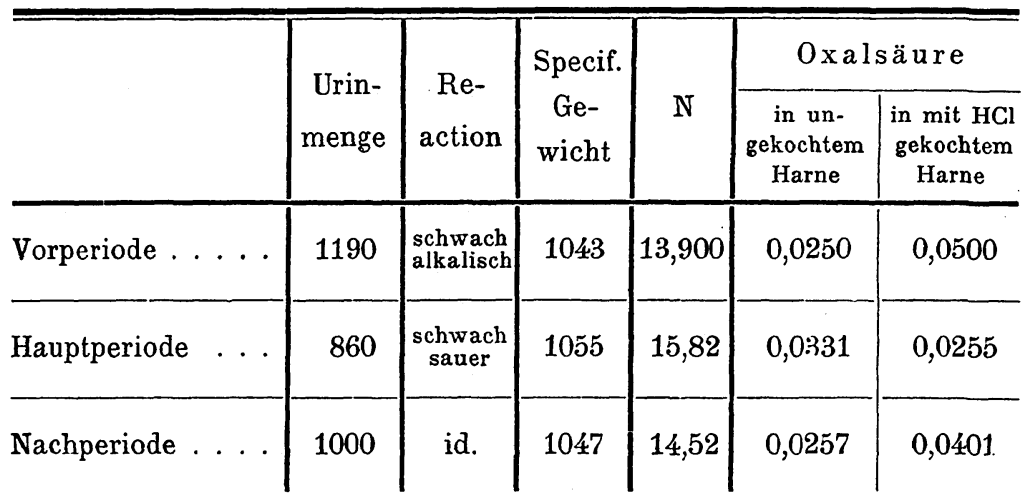

Versuch II. Ein Hund von 6 Kilo Gewicht wird während 6 Tagen mit $200 \mathrm{~g}$ Brot, $500 \mathrm{~g}$ Milch, 40 g Speck täglich gefüttert. Am 4., 5., 6. Tage wird täglich $1 / 2 \mathrm{~g}$ Harnsäure, in Piperazin gelöst, in das Unterhautgewebe eingespritzt. Jedes $1 / 2 \mathrm{~g}$ ist in $20 \mathrm{ccm}$ Wasser gelöst und die Gesammtmenge des derart eingeführten Stickstoffs beträgt 0,205 g N. Die Analyse des Urins liefert folgende Werthe:

Tabelle V.

\begin{tabular}{l|c|c|c|c|c|c}
\hline \hline & $\begin{array}{c}\text { Urin- } \\
\text { menge }\end{array}$ & $\begin{array}{c}\text { Re- } \\
\text { action }\end{array}$ & $\begin{array}{c}\text { Specif. } \\
\text { Ge- } \\
\text { wicht }\end{array}$ & $\mathrm{N}$ & $\begin{array}{c}\text { in un- } \\
\text { gekochtem } \\
\text { Harne }\end{array}$ & $\begin{array}{c}\text { in mit HCl } \\
\text { gekochtem } \\
\text { Harne }\end{array}$ \\
\hline Vorperiode ..... & 1020 & $\begin{array}{c}\text { schwach } \\
\text { sauer }\end{array}$ & 1030 & 6,76 & 0,0468 & 0,0555 \\
\hline Hauptperiode.... & 1630 & neutral & 1024 & 7,64 & 0,0470 & 0,0444
\end{tabular}

Aus äusseren Gründen musste man auf die Untersuchung einer Nachperiode verzichten.

Versuch III. Ein Kaninchen von $2300 \mathrm{~g}$ Gewicht wird während 9 Tagen mit $150 \mathrm{~g} \mathrm{Weisskohl} \mathrm{und} 150 \mathrm{~g}$ Mohrrüben gefüttert. Am 4., 5,. 6. Tage werden zur Nahrung 2,50 g Harnsäure täglich zugesetzt. Am Ende des Versuches wiegt 
das Thier $2200 \mathrm{~g}$. Aus der Urinuntersuchung erhält man folgende Werthe (wegen des geringen specifischen Gewichts des Urins wird auf die Bestimmung der Oxalursäure verzichtet und man begnügt sich, die Bestimmung im mit Salzsäure stark eingedampften Harne vorzunehmen):

Tabelle VI.

\begin{tabular}{c|c|c|c|c|c}
\hline & $\begin{array}{c}\text { Urin- } \\
\text { menge }\end{array}$ & Reaction & $\begin{array}{c}\text { Specif. } \\
\text { Gewicht }\end{array}$ & $\mathrm{N}$ & Oxalsäure \\
\hline Vorperiode..... & 730 & leicht alkalisch & 1013 & 1,89 & 0,0093 \\
\hline Hauptperiode ... & 740 & id. & 1016 & 2,48 & 0,0180 \\
\hline Nachperiode.... & 770 & id. & 1013 & 1,51 & 0,1255
\end{tabular}

Versuch IV. Ein Kaninchen von $2320 \mathrm{~g}$ Gewicht wird während 9 Tagen mit $250 \mathrm{~g}$ Weisskohl und $250 \mathrm{~g}$ Mohrrüben täglich gefüttert. Am 4., 5., 6. Tage werden mittelst der Sonde 2,50 g Harnsäure (in wenig Wasser suspendirt) eingeführt. Am Ende des Versuches ist das Gewicht des Kaninchens unverändert geblieben. Die Harnuntersuchung liefert folgende Werthe:

Tabelle VII.

\begin{tabular}{|c|c|c|c|c|c|c|}
\hline & \multirow{2}{*}{$\begin{array}{l}\text { Urin- } \\
\text { menge }\end{array}$} & \multirow{2}{*}{$\begin{array}{c}\mathrm{Re}- \\
\text { action }\end{array}$} & \multirow{2}{*}{$\begin{array}{c}\text { Specif. } \\
\text { Ge- } \\
\text { wicht }\end{array}$} & \multirow[b]{2}{*}{$\mathrm{N}$} & \multicolumn{2}{|c|}{ Oxalsäure } \\
\hline & & & & & $\begin{array}{l}\text { in un. } \\
\text { gekochtem } \\
\text { Harne }\end{array}$ & $\begin{array}{c}\text { in mit } \mathrm{HCl} \\
\text { gekochtem } \\
\text { Harne }\end{array}$ \\
\hline Vorperiode ... & 1274 & sauer & 1015 & 1,73 & $\begin{array}{l}\text { Unwågbare } \\
\text { Spuren }\end{array}$ & 0,0146 \\
\hline Hauptperiode. . . & 1460 & id. & 1012 & 3,00 & 0,0176 & 0,0125 \\
\hline Nachperiode . . & 834 & id. & 1015 & 1,41 & - & 0,0069 \\
\hline
\end{tabular}

Was die Versuche am Hunde betrifft, so ist ohne Weiteres klar, dass dabei nach Harnsäureeinführung keine Vermehrung, 
sondern eher eine Verminderung der Oxalursäure und der Oxalsäure stattgefunden hat. Am Kaninchen dagegen scheint im Versuche III eine sehr erhebliche, im Versuche IV eine minimale Vermehrung der Oxalsäure eingetreten zu sein. Ich sage absichtlich scheint, weil, meiner Meinung nach, solche Zahlen mit grosser Vorsicht verwerthet werden müssen. Zuerst ist beim Versuche III sehr auffallend, dass die höchste Vermehrung nicht in der Hauptperiode, sondern in der Nachperiode zu constatiren ist, während im Versuche IV der Unterschied zwischen Vorperiode und Nachperiode ein viel grösserer ist, als der zwischen Vorperiode und Hauptperiode; das ist ein Umstand, welcher uns die Annahme nahe legt, dass es sich um physiologische Schwankungen handeln könne. Thatsächlich konnte ich beim Kaninchen sehr hochgradige physiologische Schwankungen der Oxalsäureausscheidung constatiren, wie es z. B. durch folgenden Versuch bewiesen wird:

Ein Kaninchen wird während 9 Tagen mit $150 \mathrm{~g}$ Weisskohl und $150 \mathrm{~g}$ Mohrrüben täglich gefüttert. Das Thier wiegt am Beginne des Versuches $2445 \mathrm{~g}$, am Ende desselben $2070 \mathrm{~g}$. Der Urin wird je 3 Tage hindurch angesammelt und analysirt. Die Bestimmungen ergeben folgende Werthe:

Tabelle VIII.

\begin{tabular}{l|c|c|c|c|c|c}
\hline \hline & $\begin{array}{c}\text { Urin- } \\
\text { menge }\end{array}$ & $\begin{array}{c}\text { Re- } \\
\text { action }\end{array}$ & $\begin{array}{c}\text { Specif. } \\
\text { Ge- } \\
\text { wicht }\end{array}$ & $\mathrm{N}$ & $\begin{array}{c}\text { O x a l s ä u r e } \\
\text { in un- } \\
\text { gekochtem } \\
\text { Harne }\end{array}$ & $\begin{array}{c}\text { in mit HCl } \\
\text { gekochtem } \\
\text { Harne }\end{array}$ \\
\hline 1., 2., 3. Tag. . . . & 900 & $\begin{array}{c}\text { schwach } \\
\text { alkalisch }\end{array}$ & 1015 & 2,00 & 0,0462 & 0,0520 \\
\hline 4., 5., 6. Tag. . . & 725 & id. & 1015 & 1,62 & 0,0244 & - \\
\hline 7., 8., 9. Tag. . . & 770 & id. & 1015 & 1,58 & 0,0308 & 0,0316 \\
\hline 10., 11., 12. Tag*). & 1295 & schwach & 1015 & - & 0,0040 & 0,0167 \\
\hline
\end{tabular}

*) Diese letzte Untersuchungsperiode fand 1 Monat später unter veränderter Diät (250 g Weisskohl und $250 \mathrm{~g}$ Mohrrüben täglich) statt. 
Diese grossen physiologischen Schwankungen müssen uns in der Deutung der hohen Zahl der Nachperiode im Versuche III (Tabelle VI) als Folge der Harnsäurefütterung vorsichtig machen. Möglicher Weise liegt die Ursache der physiologischen Schwankungen in dem verschiedenen Gehalt der Futtermittel an Oxalsäure, so dass es nöthig ist, bei einem Versuche das Futter stets aus demselben Vorrath $\mathrm{zu}$ entnehmen.

Es frägt sich nun, wie man diese ausbleibende Vermehrung der Oxalsäureausscheidung bei Harnsäureeinführung deuten muss. Man darf zuerst nicht glauben, dass die Harnsäure nicht resorbirt worden ist, da die charakteristische Vermehrung des Totalstickstoffs immer rechtzeitig eintrat.

Zweitens könnte man denken, dass die Umwandlung der Harnsäure in Oxalsäure, welche in vitro so leicht vor sich geht, im Thierorganismus, wo die Verhältnisse viel verwickelter sind, aus uns unbekannten Gründen ausbleibt. Eine solche Annahme ist sicher nicht ohne Weiteres von der Hand zu weisen; trotzdem kann man dagegen einige ernste Bedenken erheben; das Nichterscheinen der Oxalsäure im Urin schliesst nämlich die vermehrte Bildung desselben nicht aus, da es wohl möglich ist, dass die neugebildete Oxalsäure weitere Umwandlungen erleidet. Es ist noch eine strittige Frage, ob die Oxalsäure im Thierorganismus weiter verbrannt wird oder nicht. Einige Autoren, wie Pohl, ${ }^{18}$ ) Gaglio, ${ }^{19}$ ) Abeles, ${ }^{20}$ ) Wiener ${ }^{21}$ ) und Faust, ${ }^{22}$ ) glauben, dass die Oxalsäure den Organismus ganz unverändert durchläuft; andere und speciell alle neueren Forscher haben dagegen eine mehr oder weniger vollständige Zerstörung dieses Körpers wiederholt constatirt (Marfori, ${ }^{23}$ ) Giunti, ${ }^{24}$ Klemperer und Tritschler, Lommel, Stradomsky, Hildebrandt, Barth und Autenrieth). Die Frage scheint also heutzutage vielmehr im Sinne der Oxydation als in der entgegengesetzten Richtung gelöst zu sein. Folglich könnte man wohl annehmen, dass die Harnsäure sich thatsächlich im Thierkörper in Oxalsäure umwandelt, dass aber die neugebildete Oxalsäure weiter oxydirt wird und deswegen nicht im Urin erscheinen kann. Ein Versuch von Klemperer und Tritschler, welche eine Umwandlung der Harnsäure in 
Oxalsäure durch frisches Blut nachgewiesen haben, kann diese Annahme wesentlich unterstützen.

Es frägt sich nun, ob nicht nur die als solche eingeführte Oxalsäure, sondern auch die Zwischenproducte zwischen Harn und Oxalsäure (Alloxan, Parabansäure, Oxalursäure) weiter oxydirt werden können. Koehne ${ }^{25}$ ) hat bewiesen, dass eine solche Oxydation für Alloxan und für Parabansäure stattfindet; ich konnte sie für das subcutan eingeführte oxalursaure Ammon nachweisen, wie aus folgendem Versuche erhellt:

Ein Hund von $6 \mathrm{~kg}$ Gewicht wird während 20 Tagen mit $200 \mathrm{~g}$ Brod, $500 \mathrm{~g}$ Milch, $40 \mathrm{~g}$ Speck täglich gefüttert. Am 6., 7., 8. Tage werden je $10 \mathrm{ccm}$, am 9., 10. Tage je $20 \mathrm{ccm}$ einer gesättigten Lösung von oxalursaurem Ammon subcutan*) eingespritzt. Die Gesammtmenge der eingeführten Oxalursäure beträgt (als Oxalsäure berechnet) $0,1316 \mathrm{~g}$ $\mathrm{CaO}=0,2114 \mathrm{~g}$ Oxalsäure. An der Stelle der Einspritzungen ist nur eine einfache, aseptische Verhärtung zu constatiren, welche im Laufe der Nachperiode fast vollständig verschwand. Die Urinbestimmungen liefern folgende Werthe:

Tabelle IX.

\begin{tabular}{|c|c|c|c|c|c|c|}
\hline & \multirow{2}{*}{$\begin{array}{l}\text { Urin- } \\
\text { menge }\end{array}$} & \multirow{2}{*}{$\begin{array}{c}\text { Re- } \\
\text { action }\end{array}$} & \multirow{2}{*}{$\begin{array}{c}\text { Specif. } \\
\text { Ge- } \\
\text { wicht }\end{array}$} & \multirow[b]{2}{*}{$\mathrm{N}$} & \multicolumn{2}{|c|}{ Oxalsäure } \\
\hline & & & & & $\begin{array}{l}\text { in un- } \\
\text { gekochtem } \\
\text { Harne }\end{array}$ & $\begin{array}{l}\text { in mit } \mathrm{HCl} \\
\text { gekochtem } \\
\text { Harne }\end{array}$ \\
\hline Vorperiode & 2080 & $\begin{array}{l}\text { schwach } \\
\text { sauer }\end{array}$ & 1025 & 12,08 & 0,0400 & 0,0985 \\
\hline Hauptperiode. . . & 2010 & id. & 1024 & 10,37 & 0,0871 & 0,0838 \\
\hline Nachperiode ... . & 1510 & id. & 1031 & 10,41 & 0,0485 & 0,0458 \\
\hline $\begin{array}{l}5 \text { Tage nach der } \\
\text { Nachperiode. . }\end{array}$ & 1800 & id. & 1030 & 11,27 & 0,0781 & 0,0925 \\
\hline
\end{tabular}

Man muss aus dem Versuch schliessen, dass auch die Oxalursäure in Oxalsäure verwandelt und nachher vollständig oxydirt wird.

*) Es wurde absichtlich vermieden, das Thier mit Oxalursäure zu füttern, da, wie gesagt, dieselbe sich in Gegenwart von Salzsäure in Oxalsäure verwandelt. 
Was die Frage der Umwandlung der Harnsäure in Oxalsäure im Thierkörper betrifft, so muss man sie, glaube ich, den vorangehenden Erörterungen zufolge, augenblicklich dahingestellt sein lassen, da wir durch einfache Urinuntersuchung nicht entscheiden können, ob Oxalsäure zwar gebildet, aber weiter oxydirt ist oder nicht gebildet ist. Nur eine eingehende Untersuchung des Blutes und der Organe (Leber) nach Harnsäurefütterung wird wahrscheinlich im Stande sein, diese Frage endgültig zu lösen.

\section{Schlussfolgerungen.}

1. Es gelingt öfters, im Harne des Hundes und des Kaninchens, und in geringen Mengen auch des Menschen, eine Substanz nachzuweisen, welche durch Kochen mit Salzsäure Oxalsäure liefert. Es handelt sich aller Wahrscheinlichkeit nach um Oxalursäure.

2. Nach Zusatz von oxalursaurem Ammon zu Menschenharnen gelingt es nicht, auch nach längerer Zeit, ein Sediment von Calciumoxalat zu constatiren. Das spricht gegen die Hypothese, welche das Eintreten von diesem Sediment mit der Anwesenheit von Oxalursäure erklärt.

3. Unter einigen, noch unbekannten Bedingungen findet man weniger Oxalsäure in mit Salzsäure gekochten als in ungekochten Harnen, ein Umstand, welcher für die Technik der Oxalsäurebestimmung praktische Bedeutung haben kann.

4. Die Harnsäureeinführung verursacht weder beim $\mathrm{Ka}-$ ninchen noch beim Hunde eine nennenswerthe Vermehrung der Oxalsäureausscheidung. Man darf aber nicht ausschliessen, dass Oxalsäure sich im Organismus aus Harnsäure bildet, da sie wohl gebildet, aber oxydirt sein könnte.

5. Oxalursäure wird im Thierkörper in Oxalsäure umgewandelt und vollständig oxydirt.

Es ist mir eine angenehme Pflicht, Herrn Professor Salkowski sowohl für die Anregung zu dieser Arbeit, wie für die freundliche Unterstützung während der Ausführung ergebenst zu danken. 


\section{Litteratur.}

1) Schunck, Proceed. of the Royal Society of London, Vol. 15, S. 250;

Vol. 16, S. 140.

2) Neubauer, Zeitschr. für analyt. Chemie, Bd. 7, S. 225.

3) Salkowski, Diese Zeitschr., Bd. XXIX, H. 4, 5. 1900.

4) Barth u. Autenrieth, Ibidem, Bd. XXXV, H. 4, 5. 1900.

5) Hildebrandt, Ibidem, Bd. XXXV, H. 2. 1900.

6) Klemperer u. Tritschler, Zeitschr. f. klin. Medicin. 1900.

7) Wöhler u. Frerichs, Annalen der Chemie und Pharmacie, Bd. 65, S. 340.1848.

8) Gallois, Comptes Rendus des l'Acad. des Sciences de Paris. Vol. 44, S. 734, Jahrg. 1857.

9) Lommel, Arch. f. klin. Medicin, Bd. 63, S. 599, Jahrg. 1899.

10) Lüthje, Zeitschr. f. klin. Medicin, Bd. 35, S. 271, Jahrg. 1898, und Bd. 39, S. 400, Jahrg. 1900.

11) Salkowski, l. c.

12) Stradomsky, Virchow's Archiv, Bd. 163, Jahrg. 1901.

13) Ne ubauer, Annal. der Chem. u. Pharm., neue Reihe, Bd. 23, S. 206, Jahrg. 1856.

14) Fürbringer, Arch. f. klin. Medicin, Bd. 18, S. 143, Jahrg. 1876.

15) Klemperer u. Tritschler, 1. c.

16) Wiener, Arch. f. experim. Pathol. u. Pharm., Bd. 42, S. 379, Jahrg. 1899.

17) Salkowski, Pflüger's Archiv, Bd. 2, S. 958.

18) Pohl, Arch. f. experim. Pathol. u. Pharm., Bd. 37, S. 415. 1896.

19) Gaglio, Ibidem, Bd. 22, S. 246. 1887.

20) Abeles, Wiener klin. Wochenschr., 1892, Nr. 19, 20.

21) Wiener, l. c.

22) Faust, Arch. f. experim. Pathol. u. Pharm., Bd. 44. 1900.

23) Marfori, Annal. di chimica e farmacologia, Bd. 25, H. 5. 1897.

24) Giunti, Ibidem, H. 10.

25) Köhne, Ueber das Verhalten einiger Säureimide im thierischen Organismus. Inaug.-Dissert. Rostock 1894. 